\title{
COMMENTARY
}

\section{Comics and Medicine: Using Graphic Narratives in Pharmacy Education}

\author{
Alexander Hoffman, PharmD, MEd \\ Northeast Ohio Medical University, Rootstown, Ohio \\ Corresponding Author: Alexander Hoffman, Northeast Ohio Medical University, 4209 St. Rt. 44, PO Box 95, Rootstown, OH \\ 44272. Tel: 330-325-6276. Email: ahoffman1@ neomed.edu
}

Submitted June 30, 2021; accepted September 3, 2021; ePublished September 2021

Keywords: graphic medicine, pharmacy education, medical humanities

\section{INTRODUCTION}

Over the last decade, interest in comics as an educational tool has increased. ${ }^{1}$ Far from the roots of their superhero origins, the artform of comics (also known as graphic novels) now includes a vast array of fictional and nonfictional storytelling. The pervasiveness of comics in popular culture is at an all-time high; in addition to blockbuster movies, comics have been adapted to television and stage, have been nominated for prestigious literary awards, and have been adopted as teaching tools across the spectrum of education. Of interest to the medical professions, a growing section of comics publishing is focused on autobiographical and medical narratives. In 2007 Williams coined the term "graphic medicine" to describe comics that embed the medical narratives of patients or caregivers to tell personal stories of illness and health. ${ }^{2}$ Graphic medicine, therefore, is a unique form of narrative medicine, an area of teaching and study that has been recognized as a way to promote healing in medical practice. Comics are a unique form of literature in that they use a multilayered language of image and text to create a meaning that neither component can separately convey. ${ }^{3}$ Graphic medical narratives have captured the attention of the medical community, leading to the development of a graphic medicine conference, and graphic medicine narratives being published in major medical journals. ${ }^{4}$ This change has opened a door to potential benefits for patient care ${ }^{5}$ as well as new arenas of knowing and self-expression for both learners and educators in health professions education. The purpose of this commentary is to familiarize readers in pharmacy education to the concepts of graphic medicine and to introduce areas where graphic medicine may be useful as a teaching tool in pharmacy curricula.

\section{Reading and Analyzing Graphic Medicine Narratives}

Including the reading and analysis of graphic medicine narratives in pharmacy education offers several specific benefits to learners. In a study by Gowda et al., using visual art helped learners in the complex interpretation of data by forcing them to use disparate points of evaluation to develop an understanding of the "whole picture." ${ }^{\text {"In graphic }}$ medicine, both image and text are involved in meaning-making, so readers must juxtapose those separate components, look for context clues, background details, and other information in order to determine what is happening on each page. Readers develop an initial impression of the page and move from that intuitive reading to a more careful examination, unveiling additional detail that helps generate a more comprehensive understanding. Using Figure 1 as an example, an initial reading shows a woman breastfeeding her child; on further inspection, detail complicates and expands the reader's understanding. An evaluation of the poetic language of the main character can be used as a measure of her mental state; combining this information with the lines under her eyes as part of an assessment of her face gives the reader an impression of mental exhaustion. The curling of the baby's cries around the mother in a speech bubble give the reader a sense of overwhelming sound, while an overflowing laundry hamper in the background leads the reader to process their living situation. The mother's body, drawn with a curving line, emphasizes the protective nature of the relationship between the mother and her infant. The author's use of dark greys in the black and white image, juxtaposed with the glowing numbers on the clock give the reader temporal context. These details give the reader information about what is happening in the narrative, but this image is further complicated by the metaphorical, as ferns and plant matter creep into the bottom panel, showing the encroachment of a dream-like state and emphasizing the mother's sleep deprivation. In addition to these individual iconographic details, readers must also consider the jump between each panel, which gives the illusion of passing time. Deep reading of a comics page is similar to deep observation of fine art, which has been shown to 
improve learners' reflection skills and observation skills. ${ }^{6}$ The process of analyzing this comics page can also be analogized to the Collect and Assess steps of the Pharmacists' Patient Care Process, ${ }^{8}$ where a pharmacist must evaluate a complex and interwoven series of data and observations, such as the patient's medication history, drug therapy regimen, health status, problem list, general appearance, and physical examination, in order to best quantify the patient's general health and medication use in order to make clinical decisions.

A second benefit to close reading of a graphic medicine narrative is the potential to implement transformative or critical pedagogies in pharmacy curricula. Mezirow's theory of transformative learning indicates that learners require a disorienting dilemma that unbalances their pre-existing internal meaning structure in order to initiate the transformative learning process. ${ }^{9}$ Graphic medicine narratives are full of stories that can potentially act as disorienting dilemmas. Recent examples include the work of cartoonist Gabby Schulz, whose graphic medicine autobiography Sick explores his experience of the United States health care system and offers a "strident critique on the entire health care system that is affordable and accessible only to the rich." ${ }^{10}$ Another is the work of Grant Jonathon, whose comics made under the nom de plume HTMLflowers discuss his diagnosis of cystic fibrosis, his perspective of health care and society as a person with a disability, and his criticisms of the Australian health care system. ${ }^{11}$ Graphic medicine narratives can be an initiating point for content reflection, leading to process and premise reflection which ultimately leads to perspective transformation. ${ }^{7}$ Furthermore, these narratives offer a centering space for critical discourse, allowing educators to act as facilitators and learners to reflect and discuss on the concerns of their daily lives vis a vis the work, thereby unveiling the nature of structural inequity in health care in various contexts.

A third benefit of the use of graphic medicine narratives in pharmacy education is their potential to engage learners on an emotional and empathetic level, in ways that text often cannot. This is of particular interest in professional identity development. Despite the inherent complexity of interpreting the meaning of a page of comics as described above, comics are seen as inherently accessible, and the development of defined iconography in comics widens their acceptability by readers. ${ }^{3,5,12}$ This function of graphic medicine narratives may now be more necessary than ever, as caregivers, learners, and educators work to process the personal and collective traumas of the COVID-19 pandemic. The use of visual outbreak narratives to "connect [caregivers] to the communities they serve and validate the personal and professional toll of the pandemic,"13 can be a helpful intermediary for learners who are slowly moving back into a less socially isolated world. The integration of graphic narratives into medical ethics coursework has also been shown to deepen student engagement with complexity of end-of-life care. ${ }^{14}$ The final goal of pharmacy education is the learner's development of both a professional competency (the skills and knowledge a pharmacist needs to practice) and a professional identity (the transformation of the layperson into a pharmacist). Graphic medicine narratives may thus help with the development of the empathy required as part of the professional identity of the pharmacist caregiver.

\section{Creating Graphic Medicine Narratives}

If reading graphic narratives offers learners in pharmacy education distinct potential benefits, the creation of those narratives offers similar but distinct benefits. The development of a graphic narrative requires two symbiotic components - the "graphics" i.e., the image on the page, and the "narrative" i.e., the story being told. Drawing, a skill that many pharmacy learners abandon after primary school, can in itself be a form of embodied learning where learners address issues of uncertainty through reflective practice and intuitive knowing. ${ }^{15}$ Likewise, the development of narrative leans into the human considerations of health care. Storytelling is a significant component of the care experience, as patients express their concerns in the form of story while caregivers both attempt to analyze that story, situate it in the patient's sociopolitical context, and develop their own "clinical story" of the patient's health and illness (or wellness). ${ }^{16}$ The combination of image and text requires learners to harness these humanistic and embodied forms of learning, expanding the pedagogical tableau.

A second potential benefit of creating graphic medicine narratives is the nurturing of creative thinking and expression. The creation, development, and refining of a story requires learners to think creatively, and the creation of images to convey that story requires the use of skills that pharmacy learners may not have mastered. Comics use a simplified style of linguistic communication, ${ }^{3}$ and creators must be able to crystalize major themes and concerns in distinct panels and pages. This requires a significant amount of creative problem solving, a skill that is essential for future pharmacists. This problem solving is enhanced by the focus on unfamiliar skills in an area where learners are likely novices. Although learners may be hesitant to create graphic medicine narratives due to a perceived lack of artistic skill, the experience of creating a graphic narrative, and the creative thinking and problem-solving used to complete this task, is far more important than the final artistic output. 
A third potential benefit of creating graphic medicine narratives is the potential development of team-orientation and team-building skills. In a fourth-year elective for medical students, Green notes that learners in his course "build camaraderie [while the process of making a graphic medicine narrative] helps them recognize that they will often need assistance to achieve important goals." "When learners are engaged in the difficult process of developing a graphic narrative, course structures and methods that encourage group participation, peer-to-peer feedback, and co-creation will facilitate this skill development. As pharmacists further integrate in to the interprofessional model of patient care in all practice settings, these skills are a critical component for professional success.

\section{CONCLUSION}

Using comics in health professions education is a new endeavor and a growing area of scholarly work. Theoretical benefits of graphic medicine have been proposed surrounding the evaluation and creation of graphic narratives. Initial research is encouraging; the use of graphic medicine narratives in clinical education settings have been shown to increase clinician empathy ${ }^{17}$ and critical reflection on communication with patients. ${ }^{17-18}$ Studies on learner perceptions have shown that comics are considered helpful as educational tools ${ }^{19}$ and their use in a medical school curriculum has facilitated better understanding of key topics in ethics and end of life care. ${ }^{14}$ Comics have also been used to teach legal concepts in pharmacy curricula. ${ }^{20}$ As the field of graphic medicine grows, situating the field's use in pharmacy education may offer learners opportunities to reflect on whole-person medical care, meaning-making, and the creative and empathetic aspects of personal and professional development that will be valuable for their future practice.

\section{ACKNOWLEDGMENTS}

The author would like to acknowledge Marnie Galloway and Grant Jonathon who graciously allowed the author to reprint their work for this commentary. 


\section{REFERENCES:}

1. Smith ST. Who Gets to Speak? The Making of Comics Scholarship. In: Graphic Medicine Manifesto. Vol 1. University Park, PA: Pennsylvania State University Press; 2015:21-40.

2. Williams, I. C. (2012). Graphic medicine: comics as medical narrative. Medical Humanities, 38(1), 21-27.

3. McCloud S. Understanding Comics: The Invisible Art. 5th ed. New York. NY: Harper Perennial: 1994.

4. Comics and Medicine Conferences. Updated February 1, 2021. Accessed May 29, 2021. https://www.graphicmedicine.org/comics-and-medicine-conferences/

5. Yu, Megan. Roles of Graphic Pathographies in Clinical Training. AMA Journal of Ethics 20.2 (2018): 115-121.

6. Gowda D, Dubroff R, Willieme A, Swan-Sein A, Capello C. Art as Sanctuary: A Four-Year Mixed-Methods Evaluation of a Visual Art Course Addressing Uncertainty Through Reflection. Acad Med. 2018;93:S8-S13.

7. Green MJ. Graphic Storytelling and Medical Narrative: The Use of Comics in Medical Education. In: Graphic Medicine Manifesto. Vol 1. University Park, PA: Pennsylvania State University Press; 2015:67-86.

8. Joint Commission of Pharmacy Practitioners. Pharmacists' Patient Care Process. May 29, 2014. Available at: https://jcpp.net/wp-content/uploads/2016/03/PatientCareProcess-with-supporting-organizations.pdf.

9. Mezirow J. Transformative learning: Theory to practice. New Directions for Adult and Continuing Education. 1997; 74: 5-12.

10. Venkatesan S, Murali C. Graphic Medicine and the Critique of Contemporary U.S. Healthcare. Journal of Medical Humanities. 2019.

11. Jonathan G. No Visitors. Vol. 1-4. Self-published. 2015-2019.

12. Squier SM. Literature and medicine, future tense: making it graphic. Lit Med. 2008 Fall;27(2):124-52.

13. Callender B, Obuobi S, Czerwiec MK, Williams I. COVID-19, comics, and the visual culture of contagion. The Lancet. 2020;396(10257):1061-1063.

14. Elghafri, AA. Integrating Comics Into Medical Ethics Education: Medical and Physician Assistant Students' Perspectives. Master's thesis. Harvard Medical School; 2017. Accessed May 28, 2021. http://nrs.harvard.edu/urn3:HUL.InstRepos:42077946

15. Lawrence, RL, ed. Bodies of Knowledge: Embodied Learning in Adult Education. New Directions for Adult and Continuing Education, Number 134. Vol. 126. San Francisco, CA: John Wiley \& Sons; 2012.

16. Charon R. Narrative Medicine: A Model for Empathy, Reflection, Profession, and Trust. JAMA. 2001;286(15):1897-1902.

17. Myers KR, George DR, Huang X, Goldenberg MD, Van Scoy LJ, Lehman E, Green MJ. Use of a graphic memoir to enhance clinicians' understanding of and empathy for patients with Parkinson disease. Perm J. 2020;24:19.060.

18. Myers KR. Graphic pathography in the classroom and the clinic: a case study. In: Graphic Medicine Manifesto. Vol 1. University Park, PA: Pennsylvania State University Press; 2015:87-114.

19. Joshi A, Hillwig-Garcia J, Joshi M, Lehman E, Khan A, Llorente A, Haidet P. Comics as an Educational Tool on a Clinical Clerkship. Acad Psychiatry. 2019;43:290-293.

20. Steinhardt SJ, Clark JE, Kelly WN, Hill AM. Active-learning Strategies for Legal Topics and Substance Abuse in a Pharmacy Curriculum. Am J Pharm Educ. 2017;25:81(1):4. 
Figure 1.
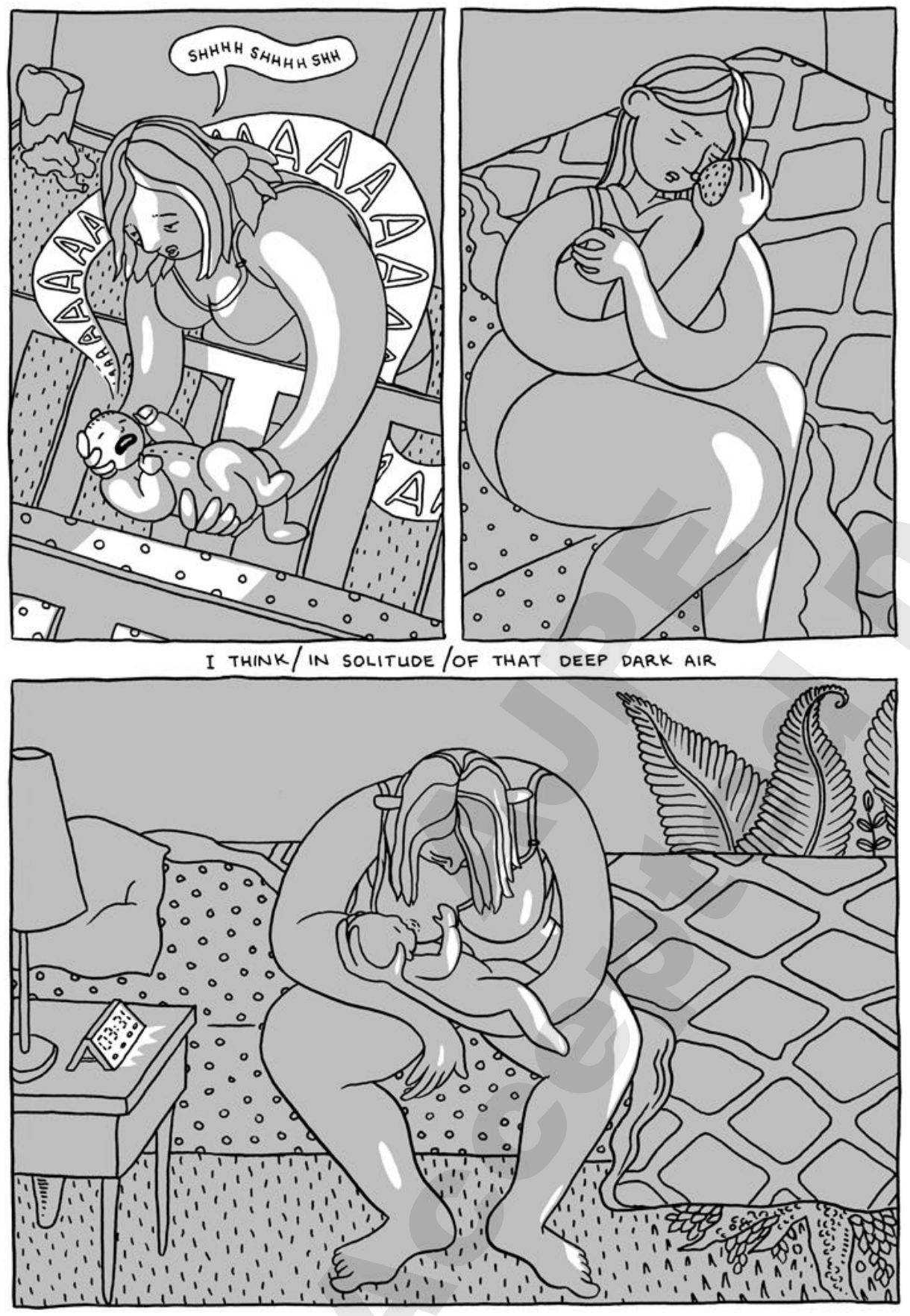

Burrow, page 17. Copyright $(2016$ by Marnie Galloway. Reprinted by permission of the author. 
Figure 2.

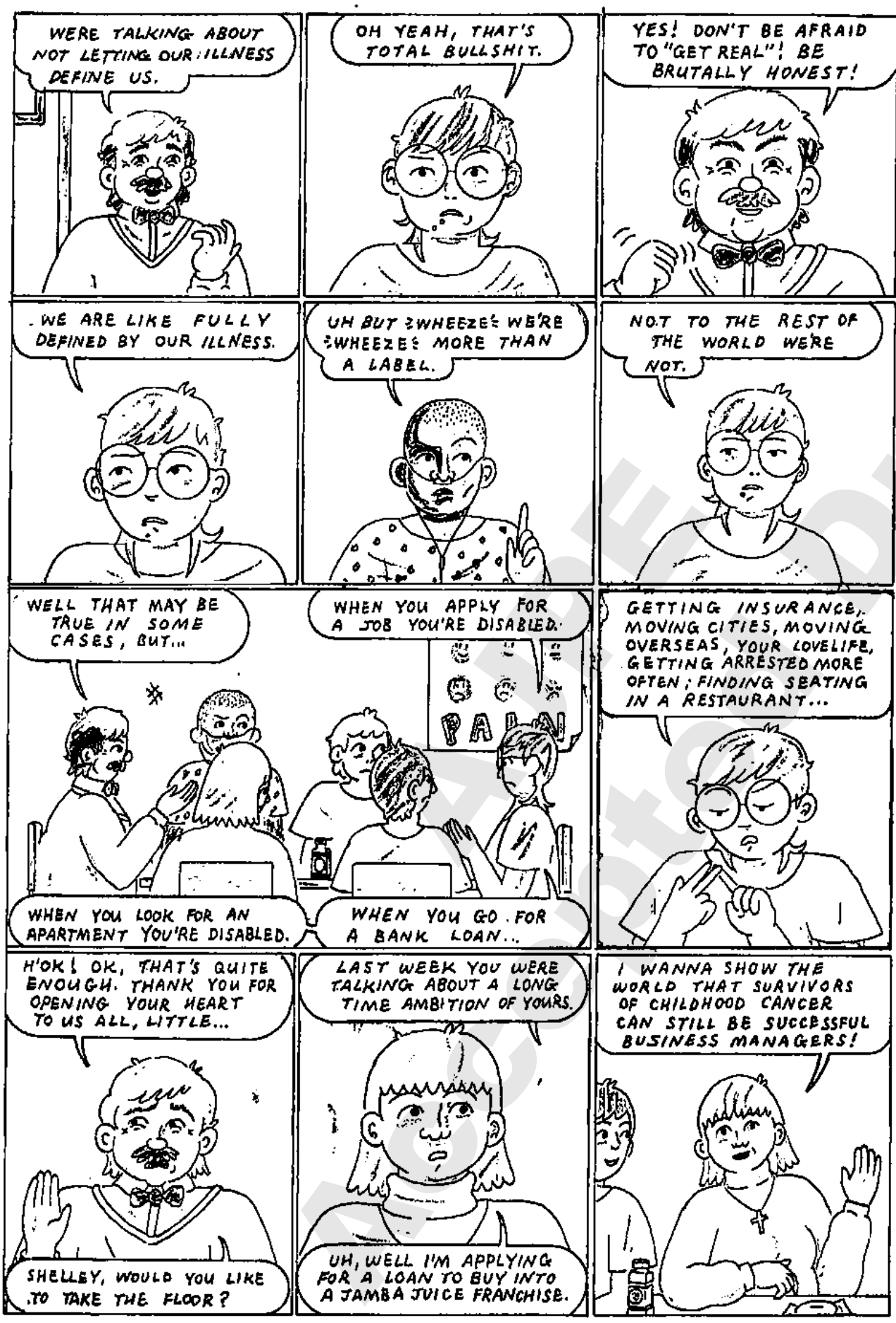

2

No Visitors Vol. 4, page 2. Copyright (C) 2019 by Grant Jonathon. Reprinted by permission of the author. In this image, the character Little (top middle panel, and throughout), acts as a stand-in for Jonathon in discussing structural discrimination against persons with disabilities. 